\title{
Normas de Vancouver
}

\section{Vancouver standards}

\section{Requisitos de uniformidad para manuscritos presentados a revistas biomédicas. 5. a edición, 1997}

\author{
Comité Internacional de Editores de Revistas Médicas*
}

* Los miembros del comité son: Linda Clever (Western Journal of Medicine), Lois Ann Colaianni (Index Medicus), Frank Davidoff (Annals of Internal Medicine), Richard Horton (Lancet), Jerome P. Kassirer y Marcia Angell (New England Journal of Medicine), George D. Lundberg y Richard Glass (Journal of the American Medical Association), Magne Nylenna (Tidsskrift for den Norske Laegeforning), Richard G. Robinson (New Zealand Medical Journal), Richard Smith (British Medical Journal), Bruce P. Squires (Canadian Medical Association Journal) y Martin Van Der Weyden (Medical Journal of Australia).

(C) 1997, Massachusetss Medical Society.

(C) de la traducción al castellano 1997, Editorial Garsi, S.A.

Un pequeño grupo de editores de revistas médicas generales se reunió informalmente en Vancouver, British Columbia, en 1978 para establecer pautas para el formato de los manuscritos presentados a estas revistas. Este grupo llegó a ser conocido como el Grupo de Vancouver. Sus requisitos pàra los manuscritos, incluyendo los formatos de referencia bibliográfica desarrollados por la Biblioteca Nacional de Medicina de los Estados Unidos, fueron publicados por primera vez en 1979. El Grupo de Vancouver creció y evolucionó hasta llegar a ser el Comité Internacional de Editores de Revistas Médicas (ICMJE), que se reúne cada año y poco a poco ha ampliado el ámbito de sus temas de preocupación.

El comité ha producido cinco ediciones de los «Requisitos de uniformidad para los manuscritos presentados a las revistas biomédicas". Con el paso de los años han aparecido temas que salen fuera de los confines de la preparación de los manuscritos. Algunos de estos temas han sido tratados en los «Requisitos uniformes»; otros han sido considerados en informes independientes. Cada informe ha sido publicado en una revista científica.

La quinta edición (1997) representa el intento de reorganizar y redactar de nuevo la cuarta edición para aumentar su claridad y examinar temas como los derechos de autor, la intimidad, las descripciones de métodos y otros asuntos. El contenido total de «Re- quisitos de uniformidad para los manuscritos presentados a las revistas biomédicas» puede reproducirse para fines didácticos y sin ánimo de lucro sin necesidad de pagar los derechos de autor; el comité desea propiciar la distribución de este material.

Las revistas que decidan seguir los «Requisitos de uniformidad» (más de 500 hasta la fecha) deben citar el documento de 1997 en sus respectivas instrucciones para autores.

Se pueden dirigir preguntas y comentarios a Kathleen Case de la secretaría del ICMJE, Annals of Internal Medicine, Colegio Americano de Médicos, Independence Mall W:, Sixth St. At Race, Philadelphia, PA 19106-1572, Estados Unidos (Teléfono: 215351-2661; Fax: 215-351-2644; correo electrónico: kathy@acp.mhs.compuserve.com).

Las publicaciones representadas en el ICMJE en 1996 eran: Annals of Internal Medicine, British Medical Journal, Canadian Medical Association Journal, Journal of the American Medical Association, Lancet, Medical Journal of Australia, New England Journal of Medicine, New Zealand Medical Journal, Tisskirft for den Norske Laegeforening, Western Journal of Medicine e Index Medicus.

Es importante subrayar tanto lo que estos requisitos implican como lo que no implican.

En primer lugar, los «Requisitos de uniformidad» son instrucciones para autores sobre la preparación de los manuscritos, no para editores sobre el estilo de publicación. (Sin embargo, muchas revistas han seguido estos requisitos en algunos de los elementos del estilo de publicación.)

En segundo lugar, si un autor prepara un manuscrito conforme al estilo especificado en estos requisitọs, los editores de las revistas participantes no devolverán el manuscrito para efectuar cambios de estilo antes de considerarlo para la publicación. No obstante, durante el proceso de publicación las revistas pueden modificar los manuscritos aceptados para que conformen sus detalles específicos al estilo de publicación respectivo. 
En tercer lugar, el autor que presenta un manuscrito a una revista participante no debe intentar prepararlo conforme al estilo de publicación de la revista, sino que debe seguir los «Requisitos de uniformidad".

Los autores también deben seguir las instrucciones para los autores de la revista en relación con los temas apropiados para la revista y los tipos de trabajos que se admiten para considerar su publicación, por ejemplo, artículos originales, revisiones o informes de casos. Por otro lado, las instrucciones de la revista probablemente indican otros requisitos propios de la revista, tales como el número de copias del manuscrito que deben presentarse, los idiomas aceptables, la extensión de los artículos y las abreviaturas autorizadas.

Se espera que las revistas participantes declaren en las instrucciones para los autores que sus requisitos se adaptan a los «Requisitos de uniformidad para los manuscritos presentados a las revistas biomédicas» y que citen una versión publicada.

\section{Consideraciones antes de presentar un manuscrito}

\section{Publicación previa o duplicada}

La publicación redundante o repetida es la publicación de un trabajo que coincide en gran medida con un trabajo ya publicado. Los lectores de las publicaciones periódicas primarias deben poder confiarse en la originalidad de lo que lean, salvo que se acompañe de una declaración clara de que el artículo está siendo publicado de nuevo a instancias del autor y el editor. Esta postura se fundamenta en el derecho internacional que regula los derechos de autor, en la conducta ética y en la utilización óptima de los recursos en relación con los costes.

La mayoría de la revistas no desea recibir manuscritos que describen un trabajo que ha sido descrito en su mayor parte en un artículo ya publicado o se contiene en otro trabajo que ha sido presentado o aceptado para publicar en otro lugar, impreso o en formato electrónico. Esta norma no impide que la revista considere un trabajo que ha sido rechazado por otra revista, o un informe completo que aparece después de haberse publicado un informe preliminar, por ejemplo un resumen o un póster expuesto en una reunión profesional. Tampoco impide que las revistas consideren un trabajo que ha sido presentado en una reunión científica pero no ha sido publicado en forma completa o está en consideración para la publicación en actas o en un formato similar. Las notas de prensa en las reuniones formales generalmente no se consideran como una violación de esta norma, pero estas notas no deben ampliarse por la aportación de más datos o de tablas y figuras.

Cuando un autor presenta un trabajo, siempre debe hacer una declaración completa al editor sobre todas las presentaciones previas e informes que pudieran interpretarse como la publicación previa o repetida del mismo trabajo o uno muy similar. El autor debe advertir al editor si el trabajo incluye sujetos que han sido incluidos en un informe previo. Cualquier trabajo precedente de este tipo debe ser citado e incluido en la bibliografia del nuevo trabajo. Se deben incluir copias de este material al presentar el trabajo nuevo para ayudar al editor a evaluar el problema.

Si se intenta hacer una publicación previa o ésta tiene lugar sin que se haya advertido al editor, los autores pueden esperar que se tomen acciones editoriales. Al menos pueden esperar que se rechace el manuscrito presentado. Si el editor no ha llegado a conocer la infracción y el artículo se publica, probablemente se insertará una nota de publicación redundante o repetida, con o sin la explicación o aprobación del autor.

La comunicación preliminar, generalmente a medios públicos, de información científica descrita en un trabajo que ha sido aceptado pero aún no ha sido publicado viola las normas de muchas revistas. En algunos casos, siempre de forma acordada con el editor, la comunicación preliminar de datos puede ser aceptable, por ejemplo, si existe un peligro para la salud pública.

\section{Publicación secundaria aceptable}

La publicación secundaria en el mismo u otro idioma, sobre todo en otros países, puede ser justificada y beneficiosa, siempre que se cumplan todas las condiciones siguientes:

- Los autores reciben la aprobación de los editores de ambas revistas; el editor implicado en la publicación secundaria debe tener una fotocopia, separata o manuscrito de la versión.

- Se respeta la prioridad de la publicación primaria por un intervalo mínimo de una semana (salvo que esto haya sido negociado específicamente por los dos editores).

- El trabajo para la publicación secundaria se destina a un grupo de lectores diferente: puede ser suficiente una versión abreviada.

- La versión secundaria refleja fielmente los datos e interpretaciones de la versión primaria.

- Un pie de página en la página titular de la versión secundaria indica a los lectores, científicos y servicios de documentación que el trabajo ha sido publicado en forma completa o parcial y proporciona la referencia primaria. Un pie de página apropiado podría ser: «Este artículo se basa en un estudio descrito por primera vez en [nombre de la revista y referencia completa]».

El permiso para este tipo de publicación secundaria debe ser gratuito. 


\section{Protección del derecho a la intimidad de los pacientes}

El derecho a la intimidad de los pacientes no debe ser infringido sin que éstos hayan dado su consentimiento informado. No se debe publicar información que permita hacer una identificación en descripciones escritas, fotografías y árboles genealógicos, salvo que esta información sea esencial para fines científicos y el paciente (o su padre o tutor) haya consentido su publicación por escrito. El consentímiento informado para esta finalidad requiere enseñar al paciente el manuscrito que ha de ser publicado.

Los detalles identificadores deben ser omitidos si no son esenciales, pero los datos de los pacientes nunca deben ser modificados o falsificados en un intento de conseguir el anonimato. El anonimato completo es difícil de lograr y se debe obtener el consentimiento informado siempre que haya alguna duda. Por ejemplo, enmascarar la región de los ojos en las fotografías de los pacientes no es suficiente para proteger el anonimato.

La exigencia de obtener el consentimiento informado debe ser incluida en las instrucciones para autores de la revista. La obtención del consentimiento informado debe constar en el artículo publicado.

\section{Requisitos para la presentación de manuscritos}

\section{Resumen de los requisitos técnicos}

- Todas las partes del manuscrito deben ser mecanografiadas o impresas a doble espacio.

- Cada sección o componente se empieza en una nueva hoja.

- La secuencia debe revisarse: página del título, resumen y palabras clave, texto, agradecimientos, referencias, tablas (cada una en una hoja) y pies de figuras.

- Las figuras (fotografiadas sin montar) no deben medir más de $203 \times 254 \mathrm{~mm}(8 \times 10$ pulgadas $)$.

- Se debe incluir la autorización para reproducir material previamente publicado o para utilizar figuras en las que se pueden identificar sujetos humanos.

- Se debe incluir la transferencia de los derechos de autor y otros formularios.

- Se debe presentar el número de copias del manuscrito exigido.

- Se debe guardar copias de todo lo que se presenta.

\section{Preparación del manuscrito}

El texto de los artículos que describen observaciones y experimentos suele (aunque no siempre) divi- dirse en secciones que llevan los títulos de Introducción, Método, Resultados y Discusión. Los artículos largos pueden precisar subtítulos para algunas secciones (sobre todo las secciones de Resultados y Discusión) para organizar su contenido. Otros tipos de artículos, tales como los informes de casos, revisiones y editoriales, pueden seguir formatos diferentes. Los autores deben consultar cada revista particularmente para obtener más información.

Se debe teclear o imprimir el manuscrito en papel blanco de $216 \times 279 \mathrm{~mm}(8,5 \times 11$ pulgadas $)$ o ISO A4 $(212 \times 297 \mathrm{~mm})$ dejando márgenes de al menos $25 \mathrm{~mm}$ (1 pulgada). El papel se teclea o imprime sólo por una cara. Se utiliza doble espacio para todo el manuscrito, incluyendo la página del título, resumen, texto, agradecimientos, referencias, tablas individuales y pies de figura. Las páginas deben numerarse consecutivamente, empezando por la página del título. El número de la página se pone en la esquina superior o inferior derecha de cada página.

\section{Manuscritos en disquete}

Cuando los trabajos están a punto de lograr la aceptación final, algunas revistas solicitan a los autores que les proporcionen una copia en formato electrónico (en un disquete); pueden aceptar archivos creados en diversos formatos de procesador de textos o en texto (ASCII).

Cuando presenten disquetes, los autores deben: asegurarse de incluir una copia impresa de la versión del artículo que está en el disquete; copiar solamente la versión más reciente del manuscrito en el disquete; nombrar claramente el archivo; etiquetar el disquete indicando el formato y nombre del archivo; y proporcionar información sobre el ordenador y programas utilizados para su creación.

Los autores deben consultar las instrucciones para autores de la revista para conocer los formatos aceptados, las convenciones para nombrar archivos, el número de copias que deben presentar y otros detalles.

\section{Primera página}

La página del título debe llevar: a) el título del artículo, que debe ser breve pero informativo; b) el nombre por el cual se conoce cada autor, con el título o títulos académicos más altos que ha obtenido y su afiliación institucional; c) el nombre de todos los departamentos e instituciones a los cuales debe ser atribuido el trabajo; d) las declaraciones que matizan la responsabilidad, si hay que hacerlas; e) el nombre y dirección del autor responsable de la correspondencia sobre el manuscrito; f) el nombre y dirección del autor a quien pueden ser solicitadas las separatas, o la notificación de que los autores no proporcionarán separatas; g) todas las fuentes de financiación en forma de 
subvenciones, equipo, fármacos, o todo lo anterior, y h) un título corto para la cabecera o pie de página, con un máximo de 40 caracteres (contando letras y espacios), en el pie de la página del título.

\section{Autoría}

Todas la personas que firman como autores deben cumplir los requisitos de autoría. Cada autor debe haber participado suficientemente en la realización del trabajo como para poder asumir una responsabilidad pública por su contenido.

La autoría debe basarse solamente en contribuciones sustantivas hechas en: a) la concepción y el diseño, o el análisis e interpretación de los datos; b) la redacción del artículo o la revisión crítica del contenido intelectual, y c) la aprobación final de la versión que va a ser publicada. Los autores deben cumplir los requisitos $a, b$ y c. Participar exclusivamente en la adquisición de recursos económicos o en la recogida de datos no justifica la autoría. La supervisión general del grupo de investigación no justifica la autoría. Cada parte del artículo que está vinculada estrechamente con las conclusiones principales debe ser la responsabilidad de al menos un autor.

Los editores pueden exigir a los autores una descripción de la aportación de cada autor, esta información puede ser publicada.

Es cada vez más frecuente la práctica de atribuir los estudios multicéntricos a un autor corporativo. Todo los miembros del grupo de autores nombrados, tanto si aparecen los nombres en el lugar reservado para los autores por debajo del título como si aparecen en un pie de página, deben cumplir plenamente los criterios de autoría descritos anteriormente. Los miembros del grupo que no cumplen estos criterios deben ser mencionados, tras obtener su autorización, en los Agradecimientos o en un apéndice (ver Agradecimientos).

El orden en que se expongan los autores debe ser una decisión tomada entre los coautores. Como el orden puede ser determinado de muchas maneras diferentes, su significado no puede ser entendido exactamente si no ha sido explicado por los autores. Los autores quizás deseen explicar los criterios que han determinado el orden de presentación de los autores en un pie de página. Cuando deciden el orden de los autores, éstos deben saber que muchas revistas limitan el número de autores incluidos en el índice del contenido y que la $\mathrm{Bi}-$ blioteca Nacional de Medicina de los Estados Unidos incluye en MEDLINE sólo los primeros 24 autores y el último autor cuando firman más de 25 autores.

\section{Resumen y palabras clave}

La segunda página debe llevar un resumen (de no más de 250 palabras para resúmenes sin estructura o 250 palabras para resúmenes estructurados). El resu- men debe comunicar el propósito del estudio o la investigación, los procedimientos básicos utilizados (selección de los sujetos del estudio o de los animales de laboratorio; métodos de observación y análisis), hallazgos principales (datos específicos con su significado estadístico si es posible) y las conclusiones más salientes. Se deben destacar los aspectos nuevos e importantes del estudio o de las observaciones.

A continuación del resumen, los autores deben proporcionar, e identificar como tales, de tres a diez palabras clave o frases cortas para ayudar a los servicios de documentación a hacer el índice cruzado del artículo, que pueden ser publicados con el resumen. Se deben utilizar términos tomados de la lista de categorías de temas médicos (medical subject headings, MeSH) del Index Medicus; si no existen términos MeSH para conceptos recientemente introducidos, pueden ser utilizados los términos utilizados en el texto.

\section{Introducción}

Se describe el propósito del artículo y las razones que han originado el estudio o la observación. Se citarán solamente las referencias relevantes y no se incluirán ni datos ni las conclusiones del trabajo.

\section{Método}

Se describe claramente la selección de los sujetos de observación o experimentación (pacientes o animales de laboratorio, incluyendo controles). Hay que identificar los sujetos por edad, sexo y otras características importantes. La definición y relevancia de la raza y etnia son ambiguas. Los autores deben ejercer cuidado especial en el uso de estas categorías.

Se identifican los métodos, aparatos (con el nombre y dirección del fabricante entre paréntesis) y procedimientos con suficiente detalle para permitir a otros trabajadores reproducir los resultados. Hay que dar referencias para los métodos establecidos, incluyendo los métodos estadísticos (ver más adelante); se ofrece la referencia y una descripción breve de los métodos que han sido publicado pero no son bien conocidos; y se describen los métodos nuevos o muy modificados, justificando su utilización y evaluando sus limitaciones. Se deben identificar precisamente todos los fármacos y productos químicos utilizados, incluyendo el nombre genérico, dosis y vía de administración.

Los informes que describen estudios clínicos aleatorios deben presentar información sobre todos los elementos principales del estudio, incluyendo el protocolo (población de estudio, intervenciones o exposiciones, resultados y la justificación del análisis estadístico), la asignación de las intervenciones (métodos de aleatoriedad, la asignación anónima del grupo de tratamiento) y el método de ocultar la identidad de los grupos (creación del grupo ciego). 
Los autores que presentan revisiones deben incluir una sección que describa los métodos utilizados para localizar, seleccionar, extraer y sintetizar datos. Estos métodos también deben ser mencionados en el resumen.

\section{Ética}

Cuando se describen experimentos que se han realizado en seres humanos, se debe indicar si los procedimientos seguidos se conformaban a las normas éticas del comité de experimentación humana responsable (institucional o regional) y a la Declaración de Helsinki de 1975, revisada en 1983 . No se deben utilizar nombres, iniciales o números de hospital, sobre todo en las figuras. Cuando se describen experimentos en animales, se debe indicar si se han seguido las pautas de una institución o consejo de investigación internacional, o una ley nacional reguladora del cuidado y la utilización de animales de laboratorio.

\section{Estadística}

Se deben describir los métodos estadísticos con suficiente detalle para permitir a un lector que tiene los conocimientos necesarios y acceso a los datos originales verificar los resultados comunicados. Cuando sea posible, conviene cuantificar los hallazgos y presentarlos con los indicadores apropiados del error de las mediciones o la incertidumbre (por ejemplo, el intervalo de confianza). Debe evitarse depender solamente de los análisis estadísticos de hipótesis, tales como el uso de valores $P$, que no transmiten una información cuantitativa importante. Se exponen los criterios de selección de los sujetos experimentales y se describen los particulares de la aleatoriedad. Hay que describir los métodos utilizados y el éxito logrado en mantener el anonimato de las observaciones. Se describen las complicaciones del tratamiento. El número de observaciones se indica y las bajas sucedidad entre las observaciones se comunican (por ejemplo, los pacientes que salen de un estudio clínico). Las referencias para el diseño del estudio y los métodos estadísticos deben hacerse a obras de consulta cuando sea posible (indicando las páginas) en lugar de los trabajos en que éstos se describieron originalmente. Se han de identificar todos los programas de ordenador de uso general utilizados.

Hay que incluir una descripción general de los métodos en la sección de Métodos. Cuando los datos son resumidos en la sección de Resultados, se especifican los métodos estadísticos utilizados para analizarlos. El número de tablas y figuras debe ser limitado a lo necesario para explicar el argumento del trabajo y facilitar la valoración de los datos en que éste se apoya. Las gráficas deben utilizarse como una alternativa a las tablas que tienen muchos apartados; no se deben duplicar datos en las gráficas y tablas. Conviene evitar el uso no técnico de los términos utilizados en estadística, tales como "aleatorio» (que implica el uso de un dispositivo de aleatoriedad), "normal», «significativo", "correlaciones" y "muestra». Hay que definir los términos, las abreviaturas y la mayoría de los símbolos.

\section{Resultados}

Los resultados se deben presentar guardando una secuencia lógica en el texto, tablas y figuras. No se debe repetir en el texto todos los datos de las tablas o de las figuras; hay que destacar o resumir sólo las observaciones importantes.

\section{Discusión}

Se destacan los aspectos nuevos e importantes del estudio y las conclusiones que han sido derivados de ellos. No se debe repetir en detalle los datos u otro material descrito en las secciones de Introducción o Resultados. La sección de Discusión debe comunicar las implicaciones y limitaciones de los hallazgos, incluyendo las implicaciones para las investigaciones en el futuro. Las observaciones se exponen en relación con otros estudios relevantes.

Las conclusiones deben ser relacionadas con los objetivos del estudio, evitando hacer declaraciones sin cualificar y conclusiones que no se apoyen completamente en los datos. Sobre todo, los autores deben evitar hacer declaraciones sobre los beneficios económicos y costes salvo que el manuscrito aporte datos y análisis económicos. Hay que evitar hablar de primicias y aludir a los trabajos que no han sido completados. Las nuevas hipótesis se describen cuando es justificado hacerlo, siempre identificándolas claramente como hipótesis. Se pueden incluir recomendaciones cuando éstas sean apropiadas.

\section{Agradecimientos}

En un lugar apropiado del artículo (el pie de página de la página del título o un apéndice al texto; consulte las instrucciones de la revista), se deben comunicar, en una o más declaraciones: a) las contribuciones que deben ser agradecidas pero no justifican la inclusión como autor, tales como el apoyo general de un jefe de departamento; b) el agradecimiento por ayuda técnica; c) el agradecimiento por ayuda económica y material, especificando la naturaleza de este apoyo, y d) las relaciones que pueden plantear un conflicto de intereses.

Las personas que han contribuido intelectualmente al trabajo, pero cuyas contribuciones no justifican la autoría pueden ser nombrados y su función o contri- 
bución descrita --por ejemplo, "consejero científico", "revisión crítica de la propuesta del estudio», "recogida de datos» o «participación en el estudio clínico». Estas personas deben haber autorizado el uso de su nombre. Los autores tienen la responsabilidad de obtener la autorización por escrito de las personas reconocidas por su nombre porque los lectores pueden inferir que ellos apoyan los datos y las conclusiones del estudio.

La ayuda técnica debe ser reconocida en un párrafo distinto de los dedicados a reconocer otras contribuciones.

\section{Referencias}

Las referencias deben ser numeradas consecutivamente en el orden en que se citan por primera vez en el texto. Las referencias se identifican en el texto, tablas y pies de figura utilizando número arábigos entre paréntesis. Las referencias citadas sólo en tablas $o$ en pies de figura deben ser numeradas de acuerdo con la secuencia establecida al identificarse por primera ve $z$ la tabla o figura correspondiente en el texto.

Se utiliza el estilo usado en los ejemplos dados a continuación, que se basan en los formatos utilizados por la Biblioteca Nacional de Medicina (NLM) de los EE.UU. en el Index Medicus. Los títulos de las revistas deben ser abreviados conforme al estilo utilizado en el Index Medicus. Consulte la List of Journals Indexed in Index Medicus, publicada anualmente como una publicación independiente de la biblioteca y como una lista recogida en el número de enero de Index Medicus. La lista también puede obtenerse en la página web de la biblioteca: http:/ / www.nlm.nih.gov.

Los resúmenes no deben utilizarse como referencias. Las referencias a trabajos aceptados pero todavía inéditos deben ser señaladas como «en prensa» o "en preparación»; los autores deben obtener autorización por escrito para citar estos trabajos y verificar que han sido aceptados para publicación. La información procedente de manuscritos presentados pero no aceptados debe ser citada en el texto como «observaciones sin publicar» con la autorización por escrito del origen.

Se debe evitar citar la "comunicación personal» salvo que ésta proporcione una información esencial no disponible en ningún recurso público, en cuyo caso se cita el nombre de la persona y la fecha de la comunicación entre paréntesis en el texto. Para los artículos científicos, los autores deben obtener la autorización por escrito y la confirmación de la certidumbre de la fuente de una comunicación personal.

Las referencias deben ser comprobadas por el autor o autores contrastándolas con los documentos originales.

El estilo de los «Requisitos uniformes» (el estilo Vancouver) se basa en su mayor parte en un estilo normativo ANSI adaptado por la NLM para sus bases de datos. Se señalan aquellos puntos donde el estilo Vancouver ahora difiere del estilo utilizado por la NLM.

\section{Artículos de revista}

\section{(1) Artículo de revista característico}

Se citan los seis primeros autores seguido por et al. (Nota: la NLM ahora cita hasta 25 autores; si hay más de 25 autores, la NLM indica los primeros 24 seguidos por el último autor $\mathrm{y}$, a continuación, et al).

Vega KJ, Pina I, Krevsky B. Heart transplantation is associated with an increased risk for pancreatobiliary disease. Ann Intern Med 1 de junio 1996;124(11):980-3.

Como una opción, si una revista utiliza la paginación continua en un volumen entero (muchas revistas médicas lo hacen), se puede ornitir el mes y el número del número. (Nota: para asegurar la uniformidad, esta opción se utiliza en todos los ejemplos de «Requisitos uniformes». La NLM no utiliza esta opción).

Vega KJ, Pina I, Krevsky B. Heart transplantation is associated with an increased risk for pancreatobiliary disease. Ann Intern Med 1996;124(11):980-3.

Cuando hay más de seis autores:

Parkin DM, Clayton D, Black RJ, Masuyer E, Friedl HP, Ivanov E, et al. Childhood leukaemia in Europe after Chernobyl: 5 year folow-up. Br J Cancer $1996 ; 73 ; 1006-12$.

\section{(2) Una organización como autor}

The Cardiac Society of Australia and New Zealand. Clinical exercise stress testing. Safety and performance guidelines. Med J Aust 1996;164:282-4.

\section{(3) Ningún autor dado}

Cancer in South Africa [editorial]. S Afr Med J 1994;84:15.

\section{(4) Artículo publicado en otro idioma}

(Nota: la NLM traduce el título, incluye la traducción en corchetes y añade una abreviatura que indica el idioma.)

Ryder TE, Haukeland EA, Solhaug JH. Bilateral infrapatellar seneruptur hos tidligere frisk kvinne. Tidsskr No Laegeforen 1996;116:41-2. 


\section{(5) Volumen con suplemento}

Shen HM, Zhang QF. Risk assessment of nickel carcinogenicity and occupational lung cancer. Environ Health Perspect 1994;102 Supl 1:275-82.

\section{(6) Núntero con suplemento}

Payne DK, Sullivan MD, Massie MJ. Women's psychological reactions to breast cancer. Semin Oncol 1996;23(1 Supl 2):89-97.

\section{(7) Volumen con parte}

Ozben T, Nacitarhan S, Tuncer N. Plasma and urine sialic acid in non-insulin dependent diabetes mellitus. Ann Clin Biochem 1995;32(Pt 3):303-6.

\section{(8) Numero con parte}

Poole GH, Mills SM. One hundred consecutive cases of flap lacerations of the leg in ageing patients. N Z Med J 1994;107(986 Pt 1):377-8.

\section{(9) Numero sin volumen}

Turan I, Wredmark T, Fellander-Tsai L. Arthroscopic ankle arthrodesis in rheumatoid arthritis. Clin Orthop 1995;(320):110-4.

\section{(10) Sin número ni volumen}

Browell DA, Lennard TW. Immunologic status of the cancer patient and the effects of blood transfusion on antitumor responses. Curr Opin Gen Surg 1993:325-33.

\section{(11) Paginación en nutmeros romanos}

Fisher GA, Sikic BI. Drug resistance in clinical oncology and hematology. Introduction. Hematol Oncol Clin North Am abril 1995;9(2):xi-xii.

\section{(12) Tipo de artículo indicado cuando es necesario}

Enzensberger W, Fischer PA. Metronome in Parkinson's disease [cárta]. Lancet 1996:347:1337.

Clement J, De Bock R. Hematological complications of hantavirus nephropathy (HVN) [resumen]. Kidney Int 19:92;42:1285.

\section{(13) Artículo con una retractación}

Garey CE, Schwarzman AL, Rise ML, Seyfried TN. Ceruloplasmin gene defect associated with epilepsy in EL mice Iretractado de Garey CE, Schwarzman AL, Rise ML, Seyfried TN. En: Nat Genet 1994;6:426-31]. Nat Genet 1995;11:104.

\section{(14) Articulo retractado}

Liou GI, Wang M, Matragoon S. Precocious IRBP gene expression during mouse development [retractado en Invest Ophthalmol Vis Sci 1994;35:3127]. Invest Ophthalmol Vis Sci 1994;35:1083-8.

\section{(15) Artículo con fe de errata publicada}

Hamlin JA, Kahn AM. Herniography in symptomatic patients following inguinal hernia repair [fe de errata publicada aparece en West J Med 1995;162:278]. West J Med 1995;162:28-31.

\section{Libros y otras monografías}

(Nota: El estilo Vancouver previo ponía una coma en lugar de un punto y coma entre la editorial y la fecha.)

\section{(16) Autor o autores particulares}

Ringsven MK, Bond D. Gerontology and leadership skills for nurses. $2^{\mathrm{a}}$ ed. Albany (NY): Delmar Publishers; 1996.

\section{(17) Uno o más editores o compitadores como autores}

Norman IJ, Redfern SJ, editores. Mental health care for elderly people. New York: Churchill Linvingstone;1996.

\section{(18) Organización cono autor y editorial}

Institute of Medicine (EE.UU). Looking at the future of the Medicaid program. Washington (DC): The Institute; 1992.

\section{(19) Capítulo de un libro}

(Nota: El estilo Vancouver previo ponía dos puntos en lugar de $\mathrm{p}$. antes de los números de página.)

Phillips SJ, Whisnant JP. Hypertension and stroke. En: Laragh JH, Brenner BM, editores. Hyperten- 
sion: pathophysiology, diagnosis, and management. $2^{a}$ ed. New York: Raven Press; 1995. p. 46578.

\section{(20) Actas de conferencia}

Kimura J, Shibasaki H, editores. Recent advances in clinical neurophysiology. Proceedings of the 10th International Congress of EMG and Clinical Neurophysiology; 15-19 de octubre de 1995; Kyoto, Japón. Amsterrdam: Elsevier; 1996.

\section{(21) Ponencia de conferencia}

Bengtsson S, Solheim BG. Enforcement of data protection, privacy and security in medical informatics. En: Lun $\mathrm{KC}_{r}$ Degoulet P, Piemme TE, Rienhoff $\mathrm{O}$, editores. MEDINFO 92. Proceedings of the 7th World Congress on Medical Informatics; 6-10 de septiembre 1992; Ginebra, Suiza. Amsterdam: North-Holland;1992. p. 1561-5.

\section{(22) Informe científico o técnico}

Emitido por la agencia que lo ha financiado/patrocinado:

Smith P, Gollady K. Payment for durable medical equipment billed during skilled nursing facility stays. Final report. Dallas (TX): Dept. of Health and Human Services (US), Office of Evaluation and Inspections: octubre de 1994 Report No.: HHSIGOEI69200860.

Emitido por la agencia que lo ha realizado:

Field MI, Tranquada RE, Feasley IC, editores. Health services research: work force and education issues. Washington: National Academy Press; 1995. Contract No.: AHCPR28942008. Patrocinado por la Agency for Health Care Policy and Research.

\section{(23) Tesis doctoral}

Kaplan SJ. Post-hospital home health care: the elderly's access and utilization [tesis doctoral]. St. Louis (MO): Washington Univ.; 1995.

\section{(24) Patente}

Larsen CE, Trip R, Johnson CR, inventores: Novoste Corporation, asignado. Methods for procedures related to the electrophysiology of the heart. US patent 5,529,067. 25 de junio 1995.

\section{Otro material publicado}

\section{(25) Artículo de periódico}

Lee G. Hospitalizations tied to ozone pollution: study estimates 50,000 admissions annually. The Washington Post; 21 de junio 1996;Sect. A:3 (co. 5).

\section{(26) Material audiovisual}

HIV+/AIDS: the facts and the future [cinta de vídeo]. St. Louis (MO): Mosby-Year Book; 1995.

\section{(27) Material legal}

Derecho público:

Preventive Health Amendments of 1993, Pub. I. No. 103-183, 107 Stat. 2226 (14 de diciembre 1993).

Proyecto de ley sin promulgar:

Medical Records Confidentiality Act of 1995 S. 1360, 104th Cong., 1st Sess. (1995).

Código de Regulaciones Federales (EE.UU.):

Informed Consent, 42 C.F.R. Sect. 441.257 (1995).

Audiencia:

Increased Drug Abuse: the Impact on the Nation's Emergency Rooms: Hearings before the Subcomm. On Human Resources and Intergovernmental Relations of the House Comm. On Government Operations, 103rd Cong., 1st Sess. (25 mayo 1993).

\section{(28) Mapa}

North Carolina. Tuberculosis rates per 100,000 population, 1990 [mapa demográfico]. Raleigh: North Carolina Dept. of Environment, Health, and Natural Resources, Div. of Epidemiology; 1991.

\section{(29) Libro de la Biblia}

The Holy Bible. King James version. Grand Rapids (MI): Zondervan Publishing House; 1995. Ruth 3:118.

\section{(30) Diccionarios y obras de consulta similares}

Stedman's medical dictionary. $26^{\mathrm{a}}$ ed. Baltimore: Williams \& Wilkins; 1995. Apraxia; p. 119-20. 


\section{(31) Material de los clásicos}

The Winter's Tale: acto 5, escena 1, líneas 13-16. The complete works of William Shakespeare. Londres: Rex; 1973.

\section{Material inédito}

\section{(32) Fn prensa}

(Nota: La NLM prefiere «en preparación» porque no todos los trabajos serán publicados.)

Leshner AI. Molecular mechanism of cocaine addiction. N Engl J Med. En prensa 1997.

\section{Material electrónico}

\section{(33) Artículo de revista en formato electrónico}

Morse SS. Factors in the emergence of infectious diseases. Emerg Infect Dis [periódico en línea] enero-marzo 1995 [citado de 5 de junio 1996];1(1):[24 pantallas]. Disponible en: URL: http://www.cdc.gov/ncidod/EID/eid.htm.

\section{(34) Monografía en formato electrónico}

CDI, clinical dermatology illustrated [monografía en CD-ROM]. Reeves JRT, Maibach H. CNEA Multimedia Group, productores, $2^{\mathrm{a}}$ ed. Versión 2.0. San Diego: CMEA; 1995.

\section{(35) Archivo de computadora}

Hemodynamics III: the ups and downs of hemodynamics [programa de ordenador]. Versión 2.2. Orlando (FL): Computerized Educational Systems; 1993.

\section{Tablas}

Se debe mecanografiar o imprimir cada tabla a doble espacio en una hoja de papel individual. Las tablas no deben presentarse en forma de fotografías. Se numeran las tablas consecutivamente en el orden de su primera citación en el texto, proporcionando un título breve para cada una. Se pone una cabecera corta o abreviada en cada columna. El material explicativo se pone en los pies de página, no en la cabecera. Deben ser explicadas en los pies de página todas las abreviaturas no convencionales que se utilizan en cada tabla. En los pies de página se deben utilizar los símbolos siguientes en esta secuencia: ${ }^{*}, t, \ddagger, \S, \mid I, \mathbb{I},{ }^{* *},+t, \ddagger \neq$, etc.
Se deben identificar las medidas estadísticas de variación tales como la desviación estándar y el error estándar de la media.

No se deben utilizar líneas para separar las celdas internas horizontales y verticales.

Asegúrese de que cada tabla se cite en el texto.

$\mathrm{Si}$ se utilizan datos procedentes de otra fuente, publicada o inédita, hay que solicitar la autorización por escrito y reconocer su origen.

La utilización de demasiadas tablas en relación con la extensión del texto puede ocasionar dificultades para la maquetación de las páginas. Se deben examinar los números de la revista a la cual se piensa presentar el trabajo para calcular el número de tablas que puede ser utilizado por cada 1.000 palabras de texto.

El editor, al aceptar un trabajo, puede recomendar el depósito en un servicio de archivo de las tablas que contienen demasiados datos de archivo para poder publicarlas, tales como el National Auxiliary Publications Service de los Estados Unidos, o que los autores aseguren su disponibilidad. En este caso, se incluye una declaración apropiada en el texto. Estas tablas deben ser presentadas para consideración con el trabajo.

\section{Ilustraciones (figuras)}

Se debe presentar el número exigido de conjuntos completos de figuras. Las figuras deben ser dibujadas profesionalmente y fotografiadas; son inaceptables los rótulos hechos a mano o escritos a máquina. En lugar de los dibujos originales, placas de radiografía $\mathrm{u}$ otro material, se deben enviar fotografias nítidas en blanco y negro reveladas en papel brillante, generalmente de $127 \times 178 \mathrm{~mm}(5 \times 7$ pulgadas) pero no más que $203 \times 254 \mathrm{~mm}$ ( $8 \times 10$ pulgadas). Las letras, números y símbolos deben ser claras y uniformes y de tamaño suficiente para que cuando se reduzcan para la publicación cada apartado sea legible. Los títulos y las explicaciones detalladas deben ponerse en los pies de figura, no en las figuras mismas.

Cada figura debe llevar una etiqueta pegada en la cara posterior que indique el número de la figura, el nombre del autor y la parte superior de la figura. No se debe escribir en la parte posterior de las figuras, ni dañarlas por el uso de sujetapapeles. Las figuras no se deben doblar ni montar en cartón.

Las fotomicrografías deben llevar una referencia de escala interna. Los símbolos, flechas y letras utilizadas en las fotomicrografías deben contrastar con el fondo.

Si se utilizan fotografías de personas, los sujetos no deben ser identificables o las imágenes deben acompañarse de la autorización por escrito para utilizar la fotografía (ver la Protección del derecho a la intimidad de los pacientes).

Las figuras deben ser numeradas consecutivamente de acuerdo con el orden en que hayan sido citadas 
por primera vez en el texto. Si una figura ha sido publicada, se debe reconocer la fuente original y presentar la autorización por escrito del propietario de los derechos de autor para reproducir el material. Se requiere la autorización con independencia de la identidad del autor o la editorial, salvo para documentos del dominio público.

Para las figuras en color, se debe determinar si la revista requiere la presentación de negativos, transparencias positivas o copias en papel. Puede ser útil para el editor acompañar las figuras por unos dibujos señalados para indicar la región que será reproducida. Algunas revistas publican figuras en color sólo si ol autor paga los costes adicionales.

\section{Pies de figura}

Los pies de figura se mecanografían o imprimen a doble espacio, en una hoja aparte, utilizando números arabigos que corresponden a la numeración de las figuras. Cuando se utilizan símbolos, flechas, números o letras para identificar distintas partes de las figuras, se debe identificar y explicar claramente cada uno en el pie de figura. Se explica la escala interna y se identifica el método de coloración utilizado en las fotomicrografías.

\section{Unidades de medida}

Las medidas de longitud, altura, peso y volumen deben darse en unidades métricas (metro, kilogramo o) litro y sus múltiplos o decimales).

Las temperaturas deben darse en grados Celsius. Las tensiones arteriales se dan en milímetros de mercurio.

Todos los valores hematológicos y bioquímicos deben comunicarse en sistema métrico siguiendo el Sistema Internacional de Unidades (SI). Los editores pueden solicitar que los autores añadan unidades alternativas, no del SI, antes de la publicación.

\section{Abreviaturas y símbolos}

Se deben utilizar sólo abreviaturas convencionales. Las abreviaturas deben evitarse en el título y en el resumen. El término completo representado por una abreviatura debe preceder su primera utilización en el texto salvo que sea una unidad de medida convencional.

\section{Envío del manuscrito a la revista}

Se debe presentar el número de copias del manuscrito requerido en un sobre de papel fuerte, protegiendo las copias y figuras con cartón, si es necesario, para que no se doblen las fotografias. Las fotografías $\mathrm{y}$ transparencias pueden introducirse en un sobre separado de papel fuerte.

Los manuscritos deben ser acompañados por una carta de presentación firmada por todos los coautores. Ésta debe incluir: a) información sobre las publicaciones previas o repetidas o la presentación en otro lugar de cualquier parte del trabajo, definida anteriormente en este documento; b) una declaración de las relaciones económicas o de otra índole que pueden suponer un conflicto de intereses; $c$ ) una declaración de que el manuscrito ha sido leído y aprobado por todos los autores, y que los requisitos de autoría enunciados anteriormente en este documento se cumplen, y que cada autor cree que el manuscrito representa un trabajo honrado; y d) el nombre, dirección y número de teléfono del autor para la correspondencia, que será responsable de comunicarse con los otros autores sobre las revisiones y la aprobación final de las galeradas. La carta debe dar información adicional que puede ayudar al editor, tal como el tipo de artículo de la revista a que corresponde el manuscrito y si el autor o autores están dispuestos a satisfacer los costes de reproducir las figuras en color.

El manuscrito debe acompañarse de copias de las autorizaciones para reproducir material publicado, utilizar figuras, comunicar informacion sobre personas identificables 0 agradecer a personas por sus contribuciones por su nombre.

\section{Modelo de la citación de los requisitos uniformes - New England Journal of Medicine}

Revistas de todo el mundo han publicado los «Requisitos uniformes para los manuscritos presentados a las revistas biomédicas' y las declaraciones que lo acompañan (y varias páginas web contienen el documento). Para citar la versión más reciente de los «Requisitos uniformes", conviene asegurarse de citar una versión publicada a partir del 1 de enero de 1997 inclusive.

\section{Información para los autores}

Estas pautas coinciden con los «Requisitos uniformes para los manuscritos presentados a las revistas biomédicas». (El documento completo aparece en $\mathrm{N}$ Engl J Med 1997;336:309-15).

\section{Manuscritos}

Se aceptan para la consideración los manuscritos que contengan material original si ni el artículo ni ninguna parte de su material esencial, tablas o figuras há sido publicada o se publicará o presentará en otro 
lugar antes de aparecer en la New England Journal of Medicine. Esta restricción no se aplica a lós resúmenes o notas de prensa publicados en relación con reuniones científicas. Los autores deben presentar al editor copias de cualquier trabajo publicado, o manuscrito en preparación o presentado en otro lugar que se relaciona con el manuscrito que se presenta para la consideración de la New England Journal of Medicine. Esta revista recomienda que no se presente más de un artículo que aborde aspectos relacionados del mismo estudio.

Se debe presentar un manuscrito original con un conjunto de figuras originales y dos copias del manuscrito completo. Los manuscritos no deben tener una extensión superior a 3.000 palabras. Se ruega a los autores que proporcionen el recuento de palabras (sin el resumen y las referencias). Se ha de utilizar papel de tamaño estándar y escribir a triple espacio todo el manuscrito. Todo el material se envía al Editor, New England Journal of Medicine, 10 Shattuck St., Boston, MA 02115-6094. Una carta de presentación firmada por todos los autores debe identificar a la persona (con la dirección y el número de teléfono) responsable de las negociaciones relacionadas con el manuscrito; la carta debe indicar claramente que el manuscrito final ha sido leído y aprobado por todos los autores y que éstos han tenido cuidado de asegurar la integridad del trabajo. El nombre de al menos una persona debe acompañar un nombre de grupo por ej., Thelma J. Smith en representación del Grupo de Porfirias de Boston. Como se ha indicado en los Requisitos uniformes (ver más arriba), firmar como autor requiere haber hecho contribuciones sustantivas a: a) la concepción y el diseño, o el análisis e interpretación de los datos; y b) la redacción del artículo o la revisión crítica del contenido intelectual. Si un estudio multicéntrico tiene más de 12 autores, o un estudio de un solo centro tiene más de ocho, cada autor debe firmar una declaración que indica que ha cumplido los criterios de autoría de los Requisitos uniformes. No se incluirán más de 12 nombres por debajo del título; los demás nombres se publicarán en un pie de página. Los agradecimientos deben limitarse en extensión a una columna del espacio de la revista y se agradecerá a cada persona sólo una vez (ver el editorial en el número del 21 de noviembre de 1991).

\section{Cartas al editor}

Ver la sección de correspondencia del New England Journal of Medicine.

\section{Conflictos de intereses}

Los autores de los artículos de investigación deben comunicar, cuando presentan el artículo, todos los acuerdos económicos que tienen con cualquier empresa cuyo producto figura prominentemente en el manuscrito o con cualquier empresa que fabrica un producto competidor. Esta información será guardada en secreto mientras el trabajo está siendo revisado y no influirá sobre la decisión editorial, pero si el artículo es aceptado para la publicación los editores generalmente consultarán a los autores sobre cómo comunicar esta información a los lectores.

Como la esencia de las révisiones y editoriales es seleccionar e interpretar la literatura, esta revista espera que los autores de artículos de este tipo no tengan ningún interés económico relacionado con una empresa (o su competidor) que fabrica un producto examinado por el artículo.

\section{Unidades de medida}

Los autores deben expresar todas las medidas en unidades convencionales, dando las unidades del Sistema Internacional (SI) en paréntesis para todo el texto. Las figuras y tablas deben utilizar unidades convencionales y dar los factores de conversión en los pies de figura o pies de página. No obstante, en conformidad con los Requisitos uniformes, los manuscritos que contienen sólo unidades SI no serán devueltos al autor sólo por ese motivo.

\section{Títulos y nombres de los autores}

El manuscrito debe acompañarse por una página que contiene el título del trabajo; los títulos deben ser cortos y descriptivos (no expositivos). Se ha de incluir también un título corto para la cabecera de menos de 40 espacios tipográficos; el nombre de cada autor, incluyendo el nombre de pila y un máximo de dos títulos académicos; el nombre del departamento e institución donde se ha llevado a cabo el trabajo; la afiliación institucional de cada autor, y el nombre y dirección del autor a quien se deben dirigir las solicitudes de separatas. Se mencionarán en esta página todas las subvenciones que deben ser agradecidas.

\section{Resúmenes}

En una hoja aparte se debe dar un resumen de una longitud máxima de 250 palabras. Este resumen debe constar de cuatro párrafos que llevan los títulos de Antecedentes, Métodos, Resultados y Conclusiones. En cada apartado respectivo se debe describir brevemente el problema abordado por el estudio, cómo ha sido realizado el estudio, los resultados más destacados y las conclusiones derivadas por los autores de los resultados. 


\section{Palabras clave}

Se deben incluir de tres a 10 palabras clave al pie de la página del resumen; éstas nos ayudarán para hacer índices del artículo y pueden ser publicados con el Resumen. Se seleccionarán los términos de las categorías de temas médicos (Medical Subject Headings) del Index Medicus.

\section{Referencias}

Las referencias deben escribirse a triple espacio y numerarse consecutivamente en el orden de su citación. Las referencias citadas por primera vez en tablas o pies de figura deben numerarse para que guarden la secuencia de las referencias citadas en el texto. El estilo de las referencias es el mismo utilizado en el Index Medicus. Se incluyen todos los autores cuando hay seis o menos; cuando hay siete o más, se nombran los primeros tres seguidos por «et al.». A continuación se ofrece una referencia de ejemplo:

1. Lahita R, Kluger J, Drayer DE, Koffler D, Reidenberg MM. Antibodies to nuclear antigens in patients treated with procainamide or acetylprocainamide. N Engl J Med 1979;301:382-5.

No son aceptables las referencias numeradas para comunicaciones personales, datos inéditos y manuscritos «en preparación» o "presentados para publicación" (ver "Autorizaciones»). Si es esencial, este material puede ser incorporado en el lugar apropiado en el texto.

\section{Tablas}

Las tablas se escriben a doble espacio y cada una debe tener un título. Si se acepta un artículo, la revista tomará las medidas necesarias para asegurar el depósito de tablas extensas que contienen datos importantes en el National Auxiliary Publications Service (NAPS); pagaremos este depósito y se añadirá un pie de página apropiado al texto. Este servicio proporciona a quienes lo solicitan microfichas o fotocopias de las tablas a un precio módico.

\section{Figuras}

Las figuras deben ser diseñadas profesionalmente. Los símbolos, rótulos y números deben ser nítidos y suficientemente grandes para que conserven su legibilidad después de reducir la figura hasta tener la anchura de una sola columna.

En la parte posterior de la figura se debe incluir el número de secuencia, el nombre del autor y la orientación apropiada (por ej., «arriba»). La figura no debe montarse en cartón. Las fotomicrografías deben ser recortadas hasta tener una anchura de $8 \mathrm{~cm}$ y las fotomicrografías electrónicas deben llevar una referencia interna de escala.

Si se utilizan fotografías de pacientes, los sujetos no deben ser identificados o la fotografía debe acompañarse por la autorización escrita para utilizar la figura. Se pueden obtener formularios de autorización del editor.

Los pies de figura deben escribirse a triple espacio en una hoja aprte y no deben ser incluidos en la figura.

Se aceptan gustosamente las figuras en color. Se deben presentar transparencias y copias en papel para estas figuras.

\section{Abreviaturas}

Con la excepción de unidades de medida, no se recomienda el uso de abreviaturas. Consulte Scientific Style and Format: The CBE Manual for Authors, Editors, and Publishers (Sexta edición. New York: Cambridge University Press, 1994) para las listas de las abreviaturas convencionales. La primera vez que aparece una abreviatura, ésta debe precederse por el término que representa.

\section{Nombres de fármacos}

Generalmente se deben utilizar nombres genéricos. Cuando se utilizan marcas de propietario en la investigación, se incluye la marca registrada entre paréntesis en la sección de Métodos.

\section{Autorizaciones}

Los materiales obtenidos de otras fuentes deben acompañarse por declaraciones por escrito del autor y de la editorial autorizando su reproducción en la New England Journal of Medicine.

Se debe obtener la autorización por escrito de al menos uno de los autores de los trabajos en prensa, datos inéditos y comunicaciones personales.

\section{Revisión y acción}

Los manuscritos son examinados por el personal de redacción y suelen remitirse a revisores externos. Recomendamos a los autores sugerir los nombres de revisores potenciales pero reservamos el derecho de hacer la selección final. Sólo será devuelta una copia de un manuscrito rechazado, generalmente en el plazo de seis semanas. Las decisiones sobre los manuscritos potencialmente aceptados pueden tardar más tiempo. 